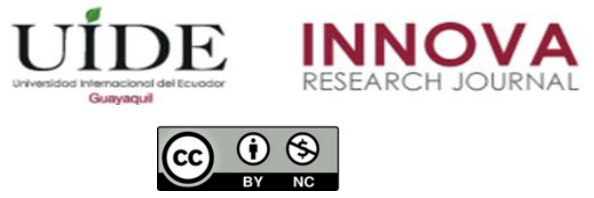

INNOVA Research Journal, ISSN 2477-9024

(Septiembre-Diciembre 2020). Vol. 5, No.3.2 pp. 97-110

DOI: https://doi.org/10.33890/innova.v5.n3.2.2020.1551

URL: http://revistas.uide.edu.ec/index.php/innova/index

Correo: innova@uide.edu.ec

\title{
Habilidades del pensamiento crítico en estudiantes de educación secundaria de Lima, Perú
}

\section{Critical thinking skills in secondary school students from Lima, Peru}

\author{
María Ysabel Álvarez Huari \\ (D) https://orcid.org/0000-0002-7831-8591 \\ Universidad Norbert Wiener, Perú \\ Isabel Menacho Vargas \\ (D) https://orcid.org/0000-0001-6246-4618 \\ Estrella Azucena Esquiagola Aranda \\ (D) https://orcid.org/0000-0002-1841-0070 \\ José Luis Camarena Mucha \\ (D) https://orcid.org/0000-0001-6133-8787 \\ Universidad César Vallejo, Perú
}

Autor para correspondencia: alvarezhmariay@gmail.com; isabelmenachov@ gmail.com; eesquiagola@ucv.edu.pe; arq.jlcamarena@gmail.com

Fecha de recepción: 21 de septiembre de 2020 - Fecha de aceptación: 14 de diciembre de 2020

\begin{abstract}
Resumen
En un mundo globalizado, donde la tecnología se desarrolla aceleradamente, es fundamental que los estudiantes de los diferentes niveles educativos desarrollen habilidades del pensamiento crítico como el razonamiento, análisis, síntesis, evaluación y toma de decisiones adecuadas para la solución de diferentes problemas, asimismo será de capaz de responder con éxito a los retos que plantea el siglo XXI. Frente a lo expuesto surge la necesidad de investigar sobre esta variable, de ahí que el objetivo del presente estudio fue describir los niveles de las habilidades del pensamiento crítico en estudiantes de Lima Metropolitana. La investigación fue desarrollada dentro del enfoque cuantitativo, tipo de investigación básica, diseño no experimental de carácter descriptivo, se trabajó con una muestra intencionada de 110 estudiantes de educación secundaria de la ciudad de Lima, Perú. Se recogió la información con el instrumento de pensamiento crítico basado en los planteamientos teóricos de Priestley (2015), el cual se dividió en veinte ítems, tres dimensiones, quince indicadores y un puntaje vigesimal. Los resultados descriptivos indicaron que el 80,0\% de participantes se ubicaron en el nivel de inicio y proceso, es decir en camino de desarrollar las habilidades cognitivas del pensamiento crítico y un 20,0\% logró aplicar de manera satisfactoria y destacada las capacidades propias de este tipo de pensamiento.
\end{abstract}

Palabras claves: pensamiento crítico; habilidades cognitivas; estudiantes de secundaria. 


\begin{abstract}
In a globalized world, where technology develops rapidly, it is essential that students at different educational levels develop critical thinking skills such as reasoning, analysis, synthesis, evaluation and adequate decision-making for the solution of different problems. Able to successfully respond to the challenges posed by the 21 st century. Faced with the above, the need to investigate this variable arises, hence the objective of this study was to describe the levels of critical thinking skills in students from Metropolitan Lima. The research was developed within the quantitative approach, type of basic research, descriptive non-experimental design, it was worked with an intentional sample of 110 secondary school students from the city of Lima, Peru. The information was collected with the critical thinking instrument based on the theoretical approaches of Priestley (2015), which was divided into twenty items, three dimensions, fifteen indicators and a vigesimal score. The descriptive results indicated that $80.0 \%$ of participants were located at the initiation and process level, that is, on the way to developing the cognitive skills of critical thinking and $20.0 \%$ managed to apply their own abilities satisfactorily and prominently of this kind of thinking.
\end{abstract}

Keywords: critical thinking; cognitive skills; high school students.

\title{
Introducción
}

En esta sociedad globalizada se hace evidente el desarrollo acelerado de la ciencia, la tecnología y las nuevas formas de comunicación, razón por la cual las escuelas han tratado de adaptarse a estos cambios, de una educación tradicional y memorístico, hemos tenido que pasar una educación por competencias, cuyo objetivo es formar estudiantes que logren desenvolverse con éxito, utilizando sus conocimientos, habilidades, actitudes y valores, en un determinado contexto, para resolver problemas que puedan presentarse en diferentes ámbitos de su vida.

Desde inicios de este siglo se viene trabajando por competencia en todas las escuelas del Perú, así como en su diferentes niveles y modalidades, sin embargo, no se está logrando esos cambios que se esperaban, muestra de ello vienen a ser los resultados de las evaluaciones naciones e internacionales en las cuales participan nuestros estudiantes peruanos. En el 2018, el Perú participa en la evaluación PISA (Programme for International Student Assessment) en las áreas de lectura, matemática y ciencia, en cada área evaluada, se definen siete a nueve niveles de desempeño ordenados en forma creciente, se considera al nivel 2 como línea base o punto de partida del desarrollo de la competencia.

La Oficina de Medición de la Calidad de los Aprendizajes (UMC) es la instancia técnica del Ministerio de Educación responsable de elaborar e implementar evaluaciones de logros de aprendizaje, además de informar los resultados. En cuanto a la participación en PISA 2018 la UMC (2019) informa que, en el área de lectura, obtuvieron una medida de promedio de 401 ubicándose por debajo de la nota promedio de 487, establecida por la Organización para la Cooperación y Desarrollo Económicos (OCDE), asimismo al realizar el análisis de los resultados se tiene que el 54, 4\% se encuentra en el nivel 1 y $-1 ; 25,8 \%$ en el $2 ; 14,3 \%$ en el 3; $4,8 \%$ en el 4; 0,08 en el 5 y ningún estudiante en el nivel 6 . Esto quiere decir que nuestros estudiantes tienen dificultad para obtener información, desarrollar una comprensión global del texto, elaborar una interpretación coherente, reflexionar y valorar el contenido y la forma del texto. 
En el área de matemática, obtuvieron una medida de promedio de 400 ubicándose por debajo de la nota promedio de 489, al realizar el análisis se tiene el 60,03\% se encuentra en el nivel 1 y -1; 23,1 en el 2; 4,1\% en el 3; 0,8 en el 5 y 0,1 en el 6. Por lo tanto, se demuestra que los estudiantes tienen dificultades para formular situaciones matemáticamente, utilizar conceptos, hechos, procedimientos y razonamiento matemáticos, así como interpretar, aplicar y evaluar los resultados matemáticos.

En el área de ciencia, obtuvieron una medida de promedio de 404 ubicándose por debajo de la nota promedio de 489, cuando se realiza el análisis se observa que el $54 \%$ se ubica en el nivel 1y -1; el 29, 2 en el 2; 13, 2 en el 3; 3,1 en el 4; 0, 2 en el 5 y ningún estudiante en el 6. En consecuencia, los estudiantes muestras dificultada para explicar fenómenos científicamente, evaluar y diseñar investigaciones científicas e interpretar datos y evidencias científicamente.

A nivel nacional, el Ministerio de Educación como todos los años aplica la evaluación censal para medir los logros de aprendizaje en segundo y cuarto grado de primaria y segundo de secundaria, esta evaluación permite conocer en qué medida los estudiantes de las instituciones educativas públicas y privadas del Perú logran los aprendizajes esperados según el Currículo Nacional de la Educación Básica (CNEB), según los promedios obtenidos los estudiantes pueden ubicarse en el nivel previo al inicio, inicio, en proceso y satisfactorio, las áreas que evalúa son: lectura, matemática y ciencia.

Según informe de la UMC (2020), en el 2019, en lectura, los participantes de segundo de secundaria obtuvieron la nota promedio de 567, ubicándolos en el nivel de inicio, quiere decir que el estudiante logró aprendizajes muy elementales respecto de lo que se espera para el ciclo evaluado en el área de lectura. Analizando tenemos que el 17,7\% de participantes se ubicaron en el nivel previo al inicio; $42,2 \%$ en inicio; $25,8 \%$ en proceso y $14,5 \%$ en satisfactorio. En consecuencia, presenta dificultad para interpretar, analizar, organizar, identificar, inferir, valorar acerca de la información del texto que lee.

En matemática, la nota promedio obtenida fue 567, es decir se ubican en el nivel inicio, indicando que logró aprendizajes muy elementales respecto de lo que se espera para el ciclo evaluado en el área de matemática, al realizar el análisis tenemos que el 33,0\% se encuentra previo al inicio; $32,1 \%$ en inicio; $17,3 \%$ en proceso y $17,7 \%$ en satisfactorio. Por lo tanto, estos resultados evidencias que muestran dificultad para resolver problemas de cantidad, regularidad, equivalencia, cambio, forma, movimiento, localización, gestión de datos e incertidumbre.

En ciencia y tecnología, la nota promedio obtenida fue 501, es decir se ubican en el nivel inicio, indicando que logró aprendizajes muy elementales respecto de lo que se espera para el ciclo evaluado en el área de ciencia y tecnología; cuando se analiza los datos, podemos señalar que el $10,0 \%$ se encuentra en previo al inicio; $43,8 \%$ en inicio; $36,3 \%$ en proceso y $9,7 \%$ en satisfactorio. Esto significa que los participantes tienen dificultades para aplicar los métodos científicos para construir sus conocimientos, explicar el mundo físico basándose en conocimientos sobre los seres vivos, materia y energía, biodiversidad, plantear soluciones tecnológicas para resolver problemas de su entorno. 
Los resultados obtenidos en las diversas evaluaciones demuestran bajo nivel de desempeño en varias habilidades cognitivas como identificar, predecir, analizar, argumentar, valorar, emitir un juicio crítico, entre otras, dichas capacidades forman parte del pensamiento crítico Özelçi y Çalışkan (2019) indican que las habilidades de pensamiento crítico mejoran la capacidad de las personas para comprender y dar sentido al mundo, los eventos y situaciones que los rodean, además de ser una de las áreas de investigación más importantes en el sistema educativo de varios países. De ahí que, Nygren, Haglund, Samuelsson, Af Geijerstam y Prytz (2019) sostienen que el pensamiento crítico debe pasar a primer plano como competencia central en sociedad actual y en los planes de estudio escolares.

Las habilidades de pensamiento crítico son habilidades que los estudiantes necesitan para enfrentar la globalización en el desafío del siglo XXI. Las habilidades de pensamiento crítico son procesos activos para pensar profundamente debido a un tema o materiales de manera significativa. Los indicadores de las habilidades de pensamiento crítico son pensar en cierto tema en profundidad, hacer preguntas y encontrar información relevante. (Sellars, Fakirmohammad, Bui, Fishetti, Niyozov, Reynolds, Ali, 2018)

Con respecto a la enseñanza del pensamiento crítico, Febri, Sajidan, Sarwanto (2019) realizaron un estudio que tuvo como objetivo analizar las habilidades del pensamiento crítico de los estudiantes de la escuela secundaria en el aprendizaje de las ciencias. Los instrumentos utilizados en este estudio se crearon de acuerdo con los indicadores de Facione sobre habilidades de pensamiento crítico, a saber, interpretar, analizar, evaluar, inferir, explicar y ejercer el autocontrol. La conclusión de este estudio fue que había dos indicadores de las habilidades de pensamiento crítico de los estudiantes que obtuvieron una buena categoría y cuatro indicadores de las habilidades de pensamiento crítico de los estudiantes obtuvieron una categoría suficiente. Por lo tanto, se necesita un seguimiento adecuado de este estudio para explorar formas de mejorar los logros de cada indicador en las habilidades de pensamiento crítico de los estudiantes.

El presente trabajo justifica su importancia por la necesidad del desarrollo integral que es el objetivo de todo sistema formal de educación. La literatura especializada ha demostrado que el desarrollo del pensamiento crítico impacta directamente en las habilidades blandas o habilidades interpersonales, como la creatividad, la resolución de conflictos, ponerse en el lugar del otro, la independencia, la autorreflexión y la adecuación a los cambios. Pensar críticamente permite aceptar los diferentes puntos de vista, establecer juicios e indicadores, para después contrastarlas y tomar en cuenta aquello que sirva para resolver determinada situación.

Frente a la realidad descrita, se propone como problema general de investigación, la siguiente interrogante ¿Cuál es nivel de pensamiento crítico en los estudiantes de educación secundaria? Asimismo, se formula como problemas específicos: ¿Cuál es nivel de pensamiento crítico en la dimensión literal en los estudiantes de educación secundaria? ¿Cuál es nivel de pensamiento crítico en la dimensión inferencial en los estudiantes de educación secundaria? ¿Cuál es nivel de pensamiento crítico en la dimensión crítico en los estudiantes de educación secundaria?

Para la presente investigación, se planteó como objetivo general: Determinar el nivel de pensamiento crítico de los estudiantes de educación secundaria en Lima. Asimismo, como 
objetivos específicos tenemos: primero, determinar el nivel de pensamiento crítico de la dimensión literal de los estudiantes de educación secundaria en Lima; segundo, determinar el nivel de pensamiento crítico de la dimensión inferencial de los estudiantes de educación secundaria en Lima y tercero, determinar el nivel de pensamiento crítico de la dimensión crítico de los estudiantes de educación secundaria en Lima.

\section{Marco Teórico}

El pensamiento crítico es un término aplicado desde la época antigua en Grecia y Roma, en cada época ha tenido diferentes enfoques y definiciones, sin embargo, en el siglo XX es cuando empieza a investigar más sobre este concepto y su importancia en la educación. Para Ennis (1991) el pensamiento crítico es un proceso cognitivo que implica reflexión, razonamiento y valoración. El trabajo científico del profesor Ennis ha sido motivación de futuras investigaciones, y en cada uno de ellos ha destacado la importancia de éste en el sistema educativo.

Fortalecer el pensamiento crítico, implica desarrollar un conjunto de actividades metodológicas, al respecto, Ennis (2005) manifiesta que el pensador crítico ideal maneja un conjunto de disposiciones y habilidades. El pensador crítico debe estar dispuesto a: ser claro en lo que pretende comunicar, ser firme en su posición, tomar en cuenta toda la situación para su evaluación, buscar y ofrecer razones fundamentadas, intentar estar informado de toda la situación, buscar alternativas razonadas y fundamentadas, buscar precisión en función a la situación analizada, practicar un pensamiento reflexivo, poseer mente abierta a los diferentes puntos de vista distinto al suyo, abstenerse de emitir juicios propios cuando las evidencias son insuficientes, evitar tomar una postura cuando las evidencias son comprobables y a utilizar las habilidades cognitivas del pensamiento crítico.

Por otro lado, Saiz y Rivas (2008) definen al pensamiento crítico como un proceso de búsqueda del conocimiento, a través de las capacidades cognitivas como la argumentación, resolución de problemas y la adecuada toma de decisiones, que conducen al logro de resultados y objetivos planificados. Asimismo, es necesario considerar que un pensador crítico y ejercitado es capaz de elaborar problemas y cuestionamientos con claridad y exactitud, evaluar información relevante y utilizar ideas abstractas para interpretarlas, llegar a conclusiones y soluciones mediante criterios o indicadores relevantes, es capaz de pensar con mente abierta, evalúa las consecuencias de diversas situaciones y comunica de manera efectiva las soluciones a los problemas encontrados (Paul y Elder, 2003).

Para Facione (2007) el pensamiento crítico es un proceso mental constituido por seis habilidades cognitivas: interpretación, análisis, evaluación, inferencia, explicación y autorregulación, la práctica de estas capacidades le permitirá al pensador crítico: despertar su curiosidad, mantenerse informado, estar alerta a las oportunidades, tener autoconfianza en sus habilidades para razonar, ser tolerante con las opiniones, ser honesto al enfrentar sus propios prejuicios y sobre todo ser prudente al plantear y valorar juicios y alternativas de soluciones. 
La capacidad de utilizar el pensamiento crítico es un objetivo importante para el cultivo del talento en el siglo XXI y es un factor intelectual, recurso que juega un papel a largo plazo y beneficia a personas de todas las edades. La capacidad de pensar críticamente es la capacidad de discernir, analizar, razonar y juzgar, así como otras actividades de pensamiento para conducir argumentos e investigación sobre situaciones, la práctica de estas actividades le permitirá los estudiantes una mejora continua en su vida académica y personal (Liu, 2019). Asimismo, se considera al pensamiento crítico como la capacidad para examinar y evaluar esta actividad cognitiva es considerada como un elemento esencial para resolver problemas, tomar decisiones y generar una actitud creativa y reflexiva (Robles, 2019).

Este estudio se basó en los trabajos realizados por Priestley (2015) quien entiende al pensamiento crítico como el procedimiento que capacita al estudiante a procesar la información. Este tipo de pensamiento se desarrolla a través de una secuencia de etapas, empezando desde la simple percepción de un hecho, objeto o fenómeno; hasta llegar a fases superiores como inferir, analizar, juzgar y plantear soluciones adecuadas a diferentes problemas que pueda encontrar. Para llegar al nivel más elevado del pensamiento crítico se debe empezar desarrollando las habilidades del nivel literal, luego el inferencial y, por último, el crítico. Estas tres etapas fueron consideradas como dimensiones y sus habilidades como indicadores del variable pensamiento crítico.

En el nivel literal se busca procesar la información a un nivel explícito. Las habilidades cognitivas que desarrolla son: observar, mirar con atención las características y cualidades de los objetos, fenómenos, personas o situaciones; discriminar, hallar diferencias básicas entre dos o más fenómenos, elementos o situaciones; identificar, reconocer detalles, establecer significados o codificar información; secuenciar, ordenar ideas, fenómenos, objetos, en función aciertos indicadores o criterios, Priestley (2015).

En el nivel inferencial, se procesa la información a nivel implícito. Las habilidades cognitivas de este nivel son: inferir; obtener nueva información a partir de información explícita; clasificar, agrupar ideas, procesos, fenómenos y objetos en función a criterios o indicadores; analizar, dividir el todo en sus partes para aclarar las relaciones entre ellas; sintetizar, señalar lo esencial o lo más importante de un texto, situación o proceso; indicar causa efecto, identificar por qué una situación o un fenómeno es efecto de otra; interpretar, expresar a un lenguaje más comprensible y coherente determinada situación; predecir, plantear hipótesis a partir de información explicita; y la resolución del problema; proponer alternativas adecuadas para solucionar una dificultad, para llegar a esta última habilidad, es necesario haber trabajado todas las anteriores, Priestley (2015).

El nivel crítico, considera como la fase superior del pensamiento crítico, porque llegar a esta fase implica haber utilizado y practicado todas las habilidades cognitivas del nivel literal e inferencial. Aquí encontramos la habilidad de argumentar, defender una posición con argumentos o razones fundamentados en un marco teórico; y juzgar, evaluar o valorar una situación, fenómeno, objeto, proceso, teniendo en cuenta un conjunto de criterios pre establecido. Dicha valoración le permitirá le permitirá tomar decisiones adecuadas, fundamentadas y elaboradas bajo un raciocinio, Priestley (2015). 
Las habilidades de pensamiento crítico son muy importantes y cada vez más necesarias debido al aumento de problemas complejos provocados por el rápido desarrollo de la tecnología y los movimientos sociales en todo el mundo. Por lo tanto, los educadores deben enseñar esas habilidades cognitivas a sus alumnos para que puedan adaptarse creativamente a nuevas situaciones, permitiéndoles el éxito a nivel personal, profesional o familiar con una participación activa como futuros ciudadanos (Changwong, Sukkamart y Sisan, 2018; Ulger, 2018).

Del mismo modo, Shirazi y Heidari (2019) frente a las puntuaciones bajas que obtuvieron sus participantes en la investigación sobre las habilidades del pensamiento crítico, plantearon que es necesario utilizar estrategias para mejorar el pensamiento crítico en esta población, incluyendo el uso frecuente de aprendizaje activo individual y grupal, capacitando a los instructores para preparar pruebas que se dirigen a altos niveles de dominio cognitivo y presentan preguntas de sondeo, alentando a los estudiantes e instructores a participar en análisis de problemas y discusiones, proporcionando diferentes ideas y opiniones, y promover el aprendizaje auto dirigido. Frente a ello, Willingham (2008), plantea que el pensamiento crítico no es solo para estudiantes avanzados, sino personas de todas las edades, las mismas que son capaces de pensar críticamente y lo utiliza todo el tiempo, inclusive desde muy pequeños; la dificultad no radica en pensar críticamente, sino en reconocer cuándo hacerlo y sabiendo lo suficiente para utilizarlo exitosamente.

\section{Metodología}

La investigación fue desarrollada dentro del enfoque cuantitativo, tipo de investigación básica, diseño no experimental de carácter descriptivo, se trabajó con una muestra intencionada de 110 estudiantes del tercer grado de educación secundaria de una Institución Educativa de Lima Metropolitana. Para recoger los datos se utilizó, como instrumento, la prueba de pensamiento crítico, basada en los planteamientos teóricos de Prietsley (2015), su objetivo fue medir el nivel de pensamiento crítico. El instrumento constó de 20 ítems, dividido en tres dimensiones y catorce indicadores; con respuestas dicotómicas y puntaje vigesimal. El instrumento fue validado por juicios de expertos y para comprobar la confiabilidad del instrumento se utilizó la técnica de Kuder Richardson (KR-20) en una muestra piloto integrada por 20 estudiantes, el resultado determinó una muy alta confiabilidad.

\section{Tabla 1}

Operacionalización de la variable pensamiento crítico

\begin{tabular}{lll}
\hline Dimensiones & \multicolumn{1}{c}{ Indicadores } & \multicolumn{1}{c}{ Niveles } \\
\hline Literal & $\begin{array}{l}\text { 1.Observar } \\
\text { 2. Discriminar }\end{array}$ & $\begin{array}{l}\text { Logro destacado: el estudiante demuestra un } \\
\text { 3ivel superior a lo esperado respecto a la } \\
\end{array}$ \\
& 4.Secuenciar u ordenar & $\begin{array}{l}\text { competencia. } \\
\end{array}$
\end{tabular}




\begin{tabular}{|c|c|c|}
\hline Dimensiones & Indicadores & Niveles \\
\hline \multirow[t]{2}{*}{ Inferencial } & $\begin{array}{l}\text { 5.Inferir } \\
\text { 6.Clasificar } \\
\text { 7.Analizar }\end{array}$ & $\begin{array}{l}\text { Logro previsto: el estudiante demuestra el } \\
\text { nivel esperado respecto a la competencia, }\end{array}$ \\
\hline & $\begin{array}{l}\text { 8.Indicar causa efecto: } \\
\text { 9 Interpretar } \\
\text { 10. Sintetizar }\end{array}$ & $\begin{array}{l}\text { En Proceso: El estudiante está en camino de } \\
\text { lograr la competencia, requiere de } \\
\text { acompañamiento. }\end{array}$ \\
\hline \multirow[t]{2}{*}{ Crítico } & $\begin{array}{l}\text { 11. Predecir } \\
\text { 12.Resolución del } \\
\text { problema }\end{array}$ & $\begin{array}{l}\text { En inicio: El estudiante demuestra un } \\
\text { avance minio en la competencia. Tiene } \\
\text { dificultades para el desarrollo de las }\end{array}$ \\
\hline & $\begin{array}{l}\text { 13.Argumentar } \\
\text { 14. Juzgar }\end{array}$ & actividades \\
\hline
\end{tabular}

\section{Resultados y discusión}

La recogida de datos nos permitió desarrollar el siguiente análisis descriptivo, a nivel de la variable de pensamiento crítico, podemos apreciar que solo el 2,7 \% de estudiantes lograron ubicarse en el nivel de logro destacado; 17,3\%, logro previsto; lo preocupante es que un 42,7\% se encuentra en proceso es decir están en camino de lograr aplicar de manera adecuada las habilidades cognitivas; y un 37,3\% está en inicio, es decir demuestra dificultad para aplicar las diferentes habilidades del pensamiento crítico. (Ver tabla 2)

\section{Tabla 2}

Distribución de frecuencia del pensamiento crítico

\begin{tabular}{lccc}
\hline & Frecuencia & Porcentaje válido & Porcentaje acumulado \\
\cline { 2 - 4 } Inicio & 41 & 37,3 & 37,3 \\
Proceso & 47 & 42,7 & 80,0 \\
Logro esperado & 17 & 15,5 & 95.5 \\
Logro destacado & 5 & 4,5 & 100,0 \\
Total & 110 & 100,0 & \\
\hline
\end{tabular}

Husna (2019) realizó una investigación experimental, en el que aplicó la pre prueba encontró que los participantes obtuvieron bajos promedios en la evaluación sobre pensamiento crítico, lo cual motivo a elaborar un programa para mejorar esos resultados, llegando a la conclusión que los profesores deben proporcionar sesiones de aprendizaje con actividades relevantes y motivadoras para que los estudiantes logren desarrollar las habilidades cognitivas del pensamiento crítico y logren tener mayor apertura y puedan aceptar el pensamiento de los demás, sin perder la capacidad de hacer justicia. 
Las habilidades de pensamiento crítico son una de las cuatro habilidades necesarias en el siglo XXI y deben enseñarse e incluirse en el plan de estudios de la educación formal. Basri, Purwanto, As'ari, Sisworo (2019) al realizar su investigación describieron las habilidades de pensamiento crítico de los estudiantes de secundaria para resolver problemas matemáticos e identificar los componentes del pensamiento crítico en las sub-habilidades de análisis, evaluación, inferencia, explicación y autorregulación. Los resultados mostraron que los estudiantes de secundaria se encontraban en una categoría baja, en especial en las subhabilidades de evaluación y análisis. Esta misma situación fue encontrada en el presente estudio, razón por la cual surge el interés por investigar cómo se puede mejorar dichos resultados.

Con respecto al papel del docente, Makhene (2019) en su investigación llegó a la conclusión, que la indagación socrática puede ser utilizada como estrategia de enseñanza y aprendizaje para facilitar el desarrollo de las habilidades de pensamiento crítico de los estudiantes caracterizado por un respeto mutuo abierto, de confianza y tolerancia. Por lo tanto, el educador necesita tomarse el tiempo adecuado para construir preguntas que inviten a la reflexión y apuntar a facilitar una discusión que sigue a un buen ejercicio de preguntas, puede utilizar las preguntas espontáneamente como una estrategia exploratoria o con contenido específico de un tema, el papel del educador es principalmente hacer preguntas y proporcionar el apoyo a los estudiantes. Es primordial y necesaria que los docentes estén preparados.

Con respecto al análisis sobre la dimensión literal, es decir aquella capacidad de lograr reconocer información de manera explícita empezando por la observación, la cual le permitirá discriminar, identificar y ordenar según criterios establecidos, los estudiantes se ubicaron con $12,0 \%$ en logro destacado, $27,0 \%$ en logro previsto; $37,0 \%$ en proceso y $24,0 \%$ en inicio (Ver tabla 3).

\section{Tabla 3}

Distribución de frecuencia de la dimensión literal del pensamiento crítico

\begin{tabular}{lccc}
\hline & Frecuencia & Porcentaje válido & Porcentaje acumulado \\
\cline { 2 - 4 } Inicio & 29 & 26,4 & 26,4 \\
Proceso & 44 & 40,0 & 66,4 \\
Logro esperado & 24 & 21,8 & 82,8 \\
Logro destacado & 13 & 11,8 & 100,0 \\
Total & 110 & 100,0 & \\
\hline
\end{tabular}

El nivel literal es el inicio o punto de partida para el desarrollo del pensamiento crítico, Priestley (2015) la denomina como la fase perceptiva porque a través de los órganos sensoriales recibiremos información para procesarla, a medida que aumenten los estímulos, la persona será capaz de observar reconocer, secuenciar, discriminar, para ello es necesario aplicar estrategias específicas para cada habilidad, porque si no se logra desarrollar estas habilidades, no se podrá llegar al análisis inferencial y menos al crítico, en ella radica su importancia y práctica. 
Con respecto al análisis sobre la dimensión inferencial, cuando el estudiante demuestra capacidad de inferir información a partir de datos explícitos, es decir es capaz de inferir, clasificar, analizar, sintetizar, reconocer la causa y el efecto de una determinada situación, interpretar, predecir y resolver problemas; los participantes se ubicaron con 8,0\% en el nivel de logro destacado, 24,0\% en logro previsto, $31,0 \%$ en proceso y un 37, $00 \%$ en inicio. Lograr los buenos resultados en esta fase implica haber desarrollado de manera eficaz las capacidades del nivel literal, recordemos que es un proceso secuencial (Ver tabla 4).

\section{Tabla 4}

Distribución de frecuencia de la dimensión inferencial del pensamiento crítico

\begin{tabular}{lccc}
\hline & Frecuencia & Porcentaje válido & Porcentaje acumulado \\
\cline { 2 - 4 } Inicio & 30 & 27,3 & 27,3 \\
Proceso & 58 & 52,7 & 80,0 \\
Logro esperado & 16 & 14,5 & 94,5 \\
Logro destacado & 6 & 5,5 & 100,0 \\
Total & 110 & 100,0 & \\
\hline
\end{tabular}

Al respecto, Mendoza, Gómez y García (2016) en su investigación experimental, en sus resultados del pos test, el grupo experimental demostró mejoras en las capacidades de explicación y deducción, con un incremento de 29,1 y 22,1\%, respectivamente en el desempeño grupal, y con un 66,6 \% y 56,2\% de mejora individual. Sin embargo, en el indicador de interpretación, inferencia y análisis no lograron notas satisfactorias, estas habilidades del nivel inferencial requieren de práctica continua y de trabajo colaborativo entre todas las áreas para que estudiante logré reconocer información implícita.

Finalmente, en el análisis sobre la dimensión crítico, el nivel más alto del pensamiento crítico en la cual el estudiante podrá argumentar y emitir juicios de valor sobre diferentes situaciones o procesos, los estudiantes se ubicaron con 4,0\% en el nivel de logro destacado, $18,0 \%$ en logro previsto, $51,0 \%$ en proceso y un $27 \%$ en inicio (Ver tabla 5)

\section{Tabla 5}

Distribución de frecuencia de la dimensión crítico del pensamiento crítico

\begin{tabular}{lccc}
\hline & Frecuencia & Porcentaje válido & Porcentaje acumulado \\
\cline { 2 - 4 } Inicio & 40 & 36,4 & 36,4 \\
Proceso & 52 & 47,3 & 83,7 \\
Logro esperado & 13 & 11,8 & 95.5 \\
Logro destacado & 5 & 4,5 & 100,0 \\
Total & 110 & 100,0 & \\
\hline
\end{tabular}

Al respecto Tosuncuoglu (2018) señala que desarrollar la capacidad de pensar críticamente es un componente vital de la enseñanza para un aprendizaje real y significativo, este proceso activo permitirá al estudiante alcanzar un objetivo y tomar una decisión adecuada, estas 
habilidades de nivel superior es lo que se espera que los estudiantes dominen, para un buen desempeño en su vida académica, personal y posteriormente laboral. Además, Siburian, Corebima, Ibrohim, y Saptasari (2019) manifiestan que empoderar las habilidades de pensamiento crítico y creativo mediante el uso de la estrategia de aprendizaje debe ser el centro de atención de educadores, investigadores y desarrolladores educativos por la gran contribución al aprendizaje cognitivo.

Asimismo, Fitriani, Asy'ari, Zubaidah y Mahanal (2019) realizaron un estudio descriptivo que tuvo como objetivo explorar el pensamiento crítico y las habilidades de análisis crítico de los futuros profesores, los resultados demostraron hay una necesidad urgente de trabajar estas competencias en los docentes, a través de planes o programas que fomenten la enseñanza del pensamiento crítico de los futuros profesores y habilidades de análisis crítico basadas en el género. Si los docentes se preparan, se garantiza una buena enseñanza sobre este tema a los estudiantes. Al respecto, Jaramillo y Naranjo (2019) sostienen que las prácticas pedagógicas deben generar y fomentar el pensamiento crítico a través de experiencias y escenarios significativos, a partir del análisis de su contexto, los estudiantes evaluarán, reflexionarán y expondrán de manera crítica sus argumentos frente a una situación problemática encontrada.

Por lo expuesto se puede indicar que surge la necesidad imperiosa de mejorar el pensamiento crítico de los estudiantes por medio de estrategias, sin embargo, al momento de seleccionar estas metodologías, es necesario tener en cuenta sus intereses y necesidades, de ahí que la tecnología se puede convertir en un buen aliado, esta generación considerada como nativos digitales lograron mejorar sus habilidades cognitivas del pensamiento crítico mediante el uso de diversas plataformas, herramientas, programas tecnológicos. (Júdex, Borjas y Torres, 2019; Le, 2019; Pérez, Delgado, Marín y Romero, 2019; Polyakova, Heiney y DeGregory, 2019).

\section{Conclusiones}

En respuesta al objetivo general de la investigación, los estudiantes de educación secundaria de Lima, se ubicaron con $80,0 \%$ en el nivel de inicio y proceso, es decir en camino de desarrollar las habilidades cognitivas del pensamiento crítico y un 20,0\% logró aplicar de manera satisfactoria y destacada las capacidades propias de este tipo de pensamiento.

En respuesta al primer objetivo específico de la investigación, los estudiantes de educación secundaria en Lima, se ubicaron con $66 \%$ en el nivel de inicio y en proceso, es decir, en camino de desarrollar las habilidades propias de la dimensión literal y solo y 33,6\% logró aplicar de manera satisfactoria y destacada las capacidades propias de esta fase.

En respuesta al segundo objetivo específico de la investigación, los estudiantes de educación secundaria en Lima, se ubicaron con 80,0\% en el nivel de inicio y en proceso, es decir en camino de desarrollar las habilidades propias de la dimensión inferencial y solo y 20,0\%\% logró aplicar de manera satisfactoria y destacada las capacidades propias de esta fase. 
En respuesta al tercer objetivo específico de la investigación, los estudiantes de educación secundaria en Lima, se ubicaron con $83,7 \%$ en el nivel de inicio y en proceso, es decir en camino de desarrollar las habilidades propias de la dimensión crítico y solo y 16,3\% \% logró aplicar de manera satisfactoria y destacada las capacidades propias de esta fase.

\section{Recomendaciones}

Frente a la investigación realizada se sugiere capacitar a los docentes en estrategias y metodologías que fomenten el pensamiento crítico, una forma constituye que lo asuman como un recurso y estrategia didáctica en la ejecución curricular, también para el desarrollo de las habilidades blandas, impregnando los temas de medio ambiente y responsabilidad social.

Sensibilizar a la comunidad educativa sobre las concepciones y aplicaciones del pensamiento crítico, que no es exclusivo de un área curricular o un nivel educativo. Debe ser aplicado desde la educación inicial en las diferentes áreas, y así sucesivamente en cada nivel incrementar el nivel de complejidad, hasta llegar a la educación superior, etapa en la que los estudiantes tendrán un alto desenvolvimiento académico frente a nuevos retos propios de su nivel educativo de cara a las exigencias contemporáneas.

\section{Referencias Bibliográficas}

Basri, H., Purwanto, As'ari, A. y Sisworo. (2019). Investigating critical thinking skill of junior high school in solving mathematical problem. International Journal of Instruction, 12(3), 745-758. https://doi.org/10.29333/iji.2019.12345a

Changwong, K., Sukkamart, A. y Sisan, B. (2018). Critical thinking skill development: Analysis of a new learning management model for Thai high schools. Journal of International Studies, 11(2), 37-48. http://dx.doi.org/10.14254/2071-8330.2018/11-2/3

Ennis, R. (2005). Pensamiento crítico: un punto de vista racional. Revista de Psicología y Educación, 11 (1), 47-64. $\quad$ Recuperado de http://www.revistadepsicologiayeducacion.es/pdf/5.pdf

Ennis, R. (1991). Critical thinking: A streamlined conception. Teaching Philosophy, 14(1), 5-25. Recuperado de https://education.illinois.edu/docs/default-source/faculty-documents/robertennis/ennisstreamlinedconception 002.pdf

Facione, P. (2007). Pensamiento Crítico: ¿Qué es y por qué es importante? Recuperado de http://eduteka.icesi.edu.co/pdfdir/PensamientoCriticoFacione.pdf

Febri, A., Sajidan, Sarwanto. (2019). Analysis of Students' Critical Thinking Skills at Junior High School in Science Learning Journal of Physics: Conference Series, 1397 (1), art. no. 012018. https://doi.org/10.1088/1742-6596/1397/1/012018

Fitriani, H., Asy'ari, M., Zubaidah, S., \& Mahanal, S. (2019). Exploring the prospective teachers' critical thinking and critical analysis skills. Journal Pendidikan IPA Indonesia, 8(3), 379390. https://doi.org/10.15294/jpii.v8i3.19434

Husna, N. (2019). Developing students' critical thinking through an integrated extensive reading program. Teflin Journal, 30(2), 212-230. Recuperado de http://journal.teflin.org/index.php/journal/article/view/727 
Jaramillo, L., y Naranjo, G. (2019). Pensamiento Crítico: Actitud Vital para Convivir. REMEA Revista Eletrônica Do Mestrado Em Educação Ambiental,36(3), 141-159. https://doi.org/10.14295/remea.v36i3.9745

Júdex J., Borjas, M., y Torres, E. (2019). Evaluación de las habilidades del pensamiento crítico con la mediación de las TIC, en contextos de educación media. Reidocrea, 8(1), 21-34. Recuperado de https://dialnet.unirioja.es/servlet/articulo?codigo=6777955

Le, N. T. (2019). How Do Technology-Enhanced Learning Tools Support Critical Thinking? Frontiers in Education. Frontiers Media S.A. https://doi.org/10.3389/feduc.2019.00126

Liu, P. (2019). Study on the Significance and Development Strategies of Critical Thinking in English Writing. Atlantis Press. https://doi.org/10.2991/icem-18.2019.89

Makhene, A. (2019). The use of the Socratic inquiry to facilitate critical thinking in nursing education. Health SA Gesondheid, 24. https://doi.org/10.4102/hsag.v24i0.1224

Mendoza, I.; Gómez, M. y García, I. (2016). El desarrollo del pensamiento crítico mediante el debate asincrónico en foros virtuales en educación secundaria. Revista Aletheia, 8(1), 100 -115. http://dx.doi.org/10.11600/21450366.8.1aletheia.80.99

Nygren, T., Haglund, J., Samuelsson, C., Af Geijerstam, А. y Prytz, J. (2019). Critical thinking in national tests across four subjects in Swedish compulsory school. Education Inquiry, 10(1), 56-75. https://doi.org/10.1080/20004508.2018.1475200

Özelçi, S. Y., y Çalışkan, G. (2019). What is critical thinking? A longitudinal study with teacher candidates. International Journal of Evaluation and Research in Education, 8(3), 495-509. https://doi.org/10.11591/ijere.v8i3.20254

Paul, R., y Elder, L. (2003). La mini-guía para el pensamiento crítico. Conceptos y herramientas. Dillon Beach, California: Fundación para el Pensamiento Crítico. Recuperado de https://www.criticalthinking.org/resources/PDF/SP-ConceptsandTools.pdf

Pérez, A., Delgado, A., Marín, P. y Romero, L. (2019). Media competence in Spanish secondary school students. Assessing instrumental and critical thinking skills in digital contexts.

Educational Sciences: Theory and Practice, 19(3), 33-48. https://doi.org/10.12738/estp.2019.3.003

Priestley, M. (2015). Técnicas y estrategias del pensamiento crítico. México: Trillas.

Polyakova, V., Heiney, S., y DeGregory, C. (2019). Using a continuing multimedia case study to develop critical thinking and empathy. Journal of Nursing Education, 58(3), 169-172. https://doi.org/10.3928/01484834-20190221-08

Robles, P. (2019). La formación del pensamiento crítico: habilidades básicas, características y modelos de aplicación en contextos innovadores. Rehuso, 4(2), 13-24. https://doi.org/10.33936/rehuso.v4i2.1725

Saiz, C. y Rivas, S. (2008). Intervenir para transferir en pensamiento crítico. Salamanca: Universidad de Salamanca.

Sellars, M., Fakirmohammad, R., Bui, L., Fishetti, J., Niyozov, S., Reynolds, R., Ali, N. (2018). Conversations on critical thinking: Can critical thinking find its way forward as the skill set and mindset of the century? Education Sciences, 8(4). https://doi.org/10.3390/educsci8040205

Shirazi, F., y Heidari, S. (2019). The relationship between critical thinking skills and learning styles and academic achievement of nursing students. Journal of Nursing Research, 27(4). https://doi.org/10.1097/jnr.0000000000000307

Siburian, J., Corebima, A. D., Ibrohim, y Saptasari, M. (2019). The correlation between critical and creative thinking skills on cognitive learning results. Eurasian Journal of Educational Research, 2019(81), 99-114. https://doi.org/10.14689/ejer.2019.81.6 
Tosuncuoglu, I. (2018). Place of critical thinking in EFL. International Journal of Higher Education, 7(4), 26-32. https://doi.org/10.5430/ijhe.v7n4p26

Ulger, K. (2018). The effect of problem-based learning on the creative thinking and critical thinking disposition of students in visual arts education. Interdisciplinary Journal of Problem-Based Learning, 12(1). https://doi.org/10.7771/1541-5015.1649

Unidad de Medición de la Calidad Educativa - UMC (2019). Evaluación PISA 2018. Recuperado de http://umc.minedu.gob.pe/wp-content/uploads/2019/12/Resultados-PISA-2018Per\%C3\%BA.pdf

Unidad de Medición de la Calidad Educativa - UMC (2020). ¿Qué aprendizajes logran nuestros estudiantes? Resultados de las evaluaciones nacionales de logros de aprendizaje 2019 Recuperado de http://umc.minedu.gob.pe/wpcontent/uploads/2020/06/Reporte-Nacional-2019.pdf

Willingham, D. T. (2008). Critical thinking: Why is it so hard to teach?Arts Education Policy Review, 109(4), 21-32, https://doi.org/10.3200/AEPR.109.4.21-32 